\title{
Image-based restoration of the concrete void system using 2D-to-3D unfolding technique
}

Yu Song a ${ }^{\text {** }}$, Chuanyue Shen ${ }^{\text {a }}$, Robbie M. Damiani ${ }^{a}$, and David A. Lange ${ }^{\text {b }}$

${ }^{a}$ Research Assistant, Dept. of Civil and Environmental Engineering, University of Illinois Urbana-Champaign, IL 61801-2352, US

${ }^{\mathrm{b}}$ Professor, Dept. of Civil and Environmental Engineering, University of Illinois UrbanaChampaign, IL 61801-2352, US

* Corresponding author:

Yu Song

Office 2145A

Newmark Civil Engineering Laboratory, MC-250

205 North Mathews Ave.

Urbana, IL 61801-2352

(217) 281-2805

Email: yusong3@illinois.edu 


\begin{abstract}
Hardened air void analysis provides essential information of concrete freeze-thaw durability based on the size and spacing of air voids in the material. As the physical freeze-thaw experiment is timeconsuming and costly, the characteristics of concrete air voids are often deemed as a proxy of the freeze-thaw performance. This analysis is typically done by measuring the $2 \mathrm{D}$ air void intersections on polished samples, but the current interpretation of the $2 \mathrm{D}$ void characters does not accurately represent the actual void structure in 3D. To solve this problem, a 2D-to-3D unfolding technique has been proposed in the field of stereology. However, the unfolding analysis is known to be sensitive to several factors, such as void population and size along with a binning scheme, where improper unfolding can considerably bias the prediction of the actual concrete void system. This study investigates the optimal strategy of conducting the unfolding analysis for concrete. The investigation is carried out on both idealized void systems to interrogate the influence of the critical factors individually, and real concrete samples with varying levels of air entrainment to assess the concrete-specific impacts. The concrete void system is studied based on a stereological model emulating the intersected 3D air voids on the surface of polished concrete. The results highlight that, for unfolding concrete voids, logarithmic binning scheme is far more accurate to linear binning. The low unfolding error of the concrete samples indicates that the proposed methodology enables an accurate restoration of 3D void size distribution.
\end{abstract}

Keywords: air void analysis, freeze-thaw, flatbed scanner, stereology, unfolding, 3D void analysis, void size distribution, lognormal distribution, logarithmic binning 


\section{Introduction}

Concrete freeze-thaw resilience is largely controlled by fine air bubbles in the cement paste. These small voids are often intentionally entrained in fresh concrete with chemical admixtures during mixing. It is commonly understood that the maximum diameter ranges between 250 to $500 \mu \mathrm{m}$ [13]. If the air is properly entrained, the concrete microstructure can be protected from stress exerted by freezing water $[4,5]$. As such, the characterization of the concrete air void system provides important information about concrete freeze-thaw durability. To date, several approaches have been developed for the void system characterization. Air content after casting can be quickly measured using an air meter on fresh concrete, while the hardened air void analysis is more credited to evaluate the void system and predict concrete freeze-thaw resilience [6,7].

The most widely accepted method for the hardened void characterization is specified in ASTM C457, which uses both air content and spacing factor to indicate the freeze-thaw protection [8]. This method was developed by Powers where a cubic lattice network with mono-sized air voids is used to model the spatial relationship between the voids and cement paste [5]. The method requires the operator to inspect the polished concrete surface under a stereomicroscope using either the linear traverse or point count approach [8]. In recent years, the conventional C457 test has been further automated with image analysis techniques using digital scans of polished concrete, implementing Powers' approach to determine spacing factor [9-15]. Meanwhile, computed tomography (CT) has made it possible to probe the actual concrete void size distribution; however, this test does not allow evaluation of a representative volume, especially for concrete, and the equipment is typically inaccessible to most practitioners [16,17].

Alternatively, the freeze-thaw protection can be investigated based on the protected paste volume (PPV) [18]. This approach assumes that each air void protects its surrounding cement paste within a certain range so that the only paste outside of the protection range suffers a high risk of freezethaw damage. The PPV analysis is usually conducted on a scanned image of a polished concrete surface [19-23]. By depicting the paste protection yielded from the air voids in the image, PPV is presented as the percentage of the paste under protection. This image-based analysis gains a more sample-specific consideration of the paste protection, as it does not assume the uniform void distribution in paste (i.e., Powers' approach), and the air void agglomeration and presence of aggregate are further accounted for. However, this approach has its limitations. As illustrated in Fig. 1, the void radius appeared in the image, $r$, does not reflect the actual sizes, $R$. This issue can considerably mislead the understanding of concrete void systems [24]. Hence, assessing the true size of the air voids is fundamental to subsequent advances in hardened air void analysis. 


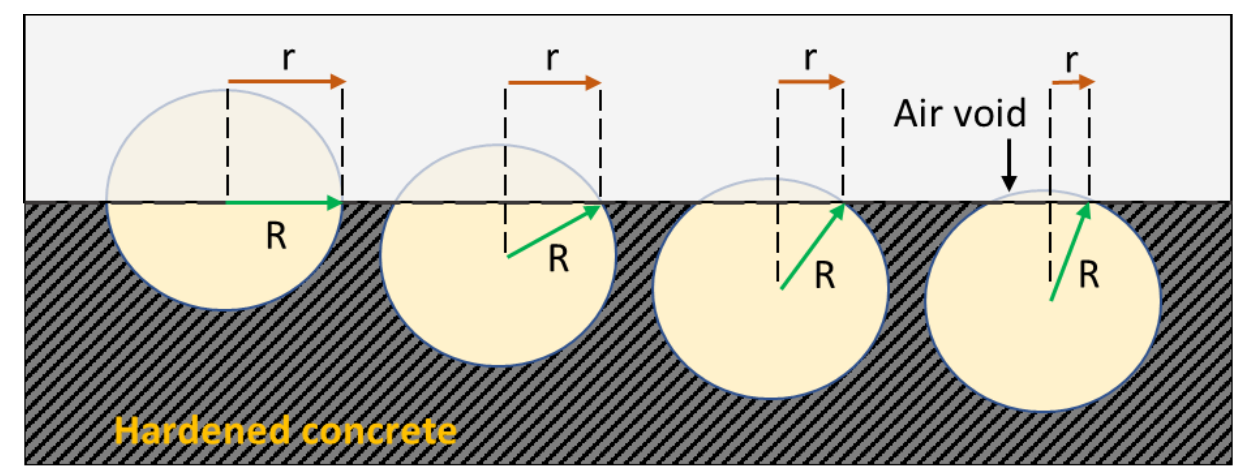

Side view of the cut section

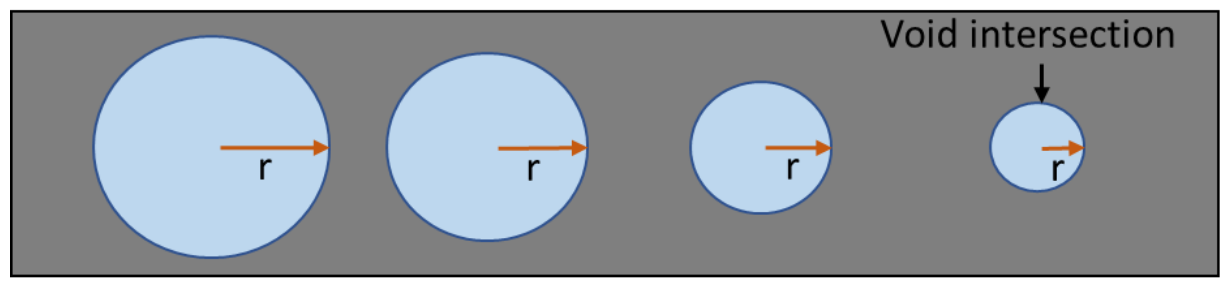

Top view on sample surface

Fig. 1: On a polished concrete surface, the apparent radius of the void intersections, $r$, on a concrete section may not reflect the actual void radius, $R$. In this case, the mono-sized air voids of $R$ yield void intersections of different $r$ when inspecting from the sample surface.

To evaluate the 3D air voids from the 2D information, a stereological technique known as 2D-to3D unfolding has been proposed [24]. The use of the unfolding technique allows one to predict the size distribution of the true voids in $3 \mathrm{D}$ with the sole knowledge of their $2 \mathrm{D}$ intersections from a scanned image. This technique offers a practical solution to access the size distribution of the 3D voids in real concrete. Although this can be arguably done with CT, it is challenging to scan a fullsize sample (e.g. $100 \mathrm{~mm}$ diameter $\times 200 \mathrm{~mm}$ height standard cylinder) with a resolution in a couple of microns to resolve the smallest air-entrained bubbles. But such a task can be accomplished using 2D imaging techniques such as the flatbed scanner method [14,19]. Nonetheless, the accuracy of the unfolding analysis can be strongly affected by several parameters, such as void population, void size, as well as image resolution [25,26]. As a result, improper unfolding can bias the air void analysis. Due to the large variation of void systems across different cases, determining a concrete-specific unfolding strategy is critical to ensure accurate prediction. In this regard, although several studies have implemented the unfolding algorithm to infer the 3D concrete void system [27,28], minimal attention is placed on how to optimize the unfolding strategy for concrete.

The objective of this work is to investigate the optimal strategy for unfolding air voids in concrete, to ensure a statistically robust prediction of the actual 3D void size distribution. In particular, the goal is to obtain the best set of unfolding results with limited information from the concrete scan 
by testing various schemes. The investigation is divided into two sections. The first section revolves around unfolding idealized void systems containing mono- and multi-sized spheres, wherein the influence of several critical parameters (e.g., void population, size, and bin number) affecting the accuracy are evaluated individually. The results from this section clarify the inherent error associated with the unfolding algorithm, providing an accuracy baseline for the subsequent analysis on concrete. The second section aims at assessing the unfolding accuracy of concrete. Three concrete mixtures exemplifying different concrete air void systems (non-, moderately-, and overly-air-entrained) are investigated for studying the concrete-specific unfolding accuracy. A stereological model is proposed to emulate the intersections between $3 \mathrm{D}$ air voids and the 2D concrete surface appeared in the scanned image. The use of this model enables interpreting the unfolding accuracy over a large area of concrete that is statistically meaningful, which would be otherwise challenging. The analysis highlights the special importance of the logarithmic binning scheme for optimal unfolding results, as compared to the conventional linear binning. Meanwhile, other practical guidelines are provided for the optimal restoration of the 3D void system from a concrete image.

\section{Background and accuracy of the unfolding algorithm}

\subsection{The 2D-to-3D unfolding technique}

Unless cutting through the centroid, a planar intersection of a sphere does not reflect the real sphere size in 3D. However, when numerous mono-sized spheres are randomly intersected, the 2D size distribution of their intersections follows a probabilistic distribution. In turn, the 3D size of these voids can be also calculated based on the 2D statistics. In stereology, this analysis is named as 2Dto-3D unfolding - a probabilistic approach to restore the 3D sizes of objects based on the statistical distribution of their 2D sizes that appeared in an image [24,29]. After including some further assumptions, the unfolding analysis can be further extended to a system containing multi-sized voids. By simplifying the concrete voids into a multi-sized sphere system, the actual concrete void size distribution can be unfolded as well [27]. Before the unfolding voids in real concrete, the unfolding accuracy and its influencing factors on idealized void systems are addressed first in the subsections.

\subsection{Unfolding accuracy for idealized void distributions}

For an isotropic uniform random (IUR) sphere system, the probability of the circular intersections falling into a size range (i.e. size bin) can be expressed by Eq. 1 [24]:

$$
\mathrm{P}\left(r_{1}<r<r_{2}\right)=\left(\sqrt{R^{2}-r_{1}^{2}}-\sqrt{R^{2}-r_{2}^{2}}\right) / R
$$

where $R$ is the sphere radius, $r$ is the range of interest, and $r_{1}$ and $r_{2}$ are the limits of the size bin. The air void system in concrete is fairly ideal in terms of the void size, shape, and distribution, which is especially true for the entrained air voids. Meanwhile, this unfolding analysis is known 
to be sensitive to the different void systems (e.g. regarding the sphere size and choice of size bins) [25]. Therefore, investigating the idealized void systems avoids the unnecessary complexity involved with real concrete. Doing so allows the ability to i) gain a specific understanding of the different influence factors, ii) optimize these factors accordingly when possible, and iii) obtain an accuracy baseline for subsequent investigation on concrete voids. Herein, the unfolding algorithm technique was encoded within a program script to automate the analysis for both mono- and multisized void systems.

A simulated mono-sized void system was investigated first by comparing the unfolding result with the known actual void population. Based on Eq.1, a 2D void size distribution from randomly intersecting one hundred $100-\mu \mathrm{m}$ radius spheres, a typical size of entrained air in concrete, and the corresponding unfolding result are illustrated in Fig. 2. Based on the analysis, the predicted void population at the actual size is 105.1. The unfolding error can be defined with Eq. 2:

$$
\mathrm{E}=\frac{\left|C_{r}-C_{t}\right|}{C_{t}} \times 100 \%
$$

where $E$ is the unfolding error, $C_{r}$ is the restored void count, and $C_{t}$ is the true count. In this case, the unfolding error is $5.1 \%$. The additional counts at the $100-\mu \mathrm{m}$ bin should be owed to the roundoff error in the other bins, where some small non-zero counts can be seen. In practice, this kind of round-off error is often related to the finite image resolution or the fact that the investigated void population is not statistically sufficient to be perfectly representative.

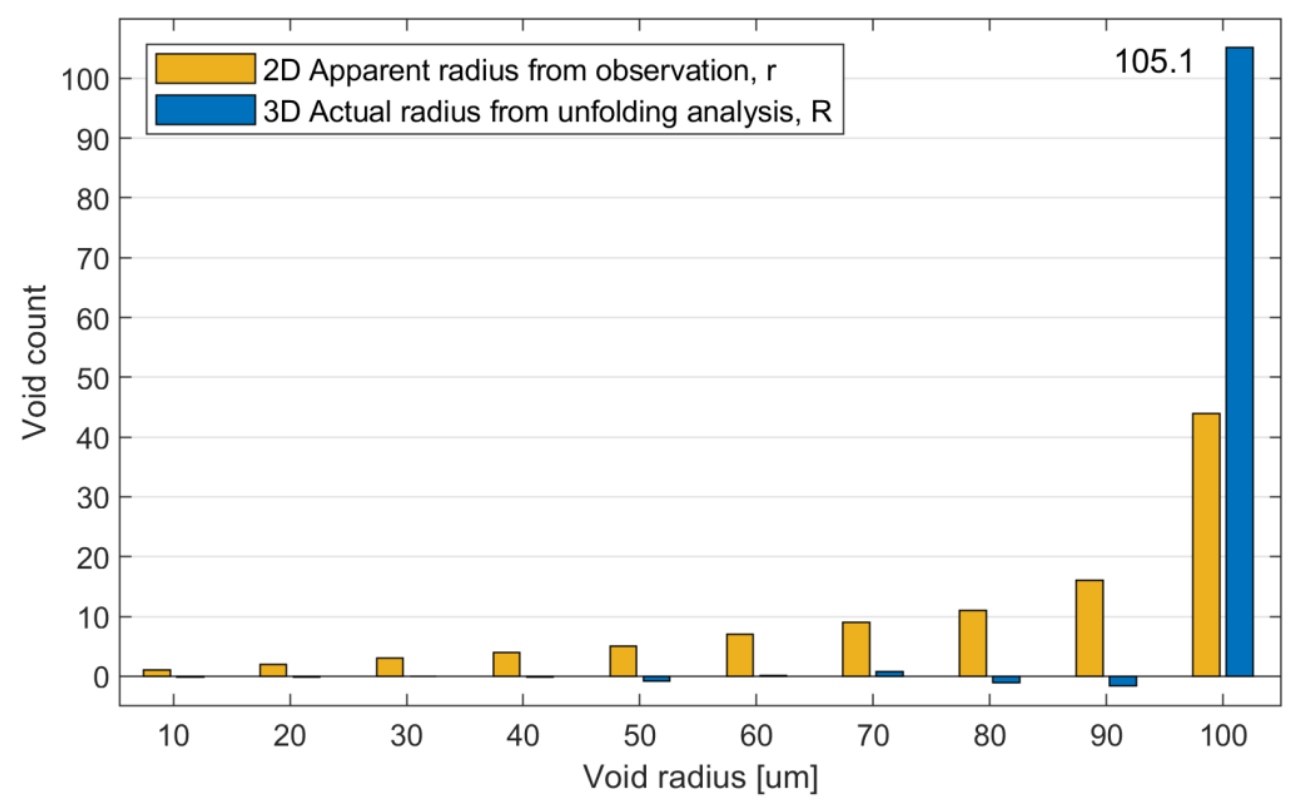

Fig. 2: Unfolding an idealized mono-sized void system $(\mathrm{R}=100 \mu \mathrm{m})$ that emulates a simplified case of one hundred entrained air voids of this size being randomly intersected on a polished concrete surface. Based on the analysis, the unfolding error of this case is $5.1 \%$. 
To more closely emulate the air system in concrete, the multi-sized void system is further analyzed. The 2D size distribution of a multi-sized void system can be treated as a superposition of multiple mono-sized distributions. As such, the unfolding can be done by sequentially processing from the large to small void. In Fig. 3, the unfolding analysis of a void system containing three typical sizes of entrained air in concrete $(30,50$, and $100 \mu \mathrm{m})$ is displayed. Here, two hundred counts were assumed for each size, and a total of twenty bins is used to present the data. The restored counts and the corresponding errors for the three sizes are 210.7 (5.4\%), 205.7 (2.9\%), and 202.3 (1.2\%), respectively. The overall unfolding error for an n-size void system is further defined in Eq. 3:

$$
\mathrm{E}=\frac{\sum_{i=1}^{n}\left|C_{r}^{i}-C_{t}^{i}\right|}{\sum_{i=1}^{n} C_{t}^{i}} \times 100 \%
$$

which results in an overall unfolding error of 3.1\% here. As compared with the mono-sized void system in Fig. 2, doubling the population for the 100- $\mu \mathrm{m}$ void in Fig. 3 decreases the error from $5.1 \%$ down to $1.2 \%$. Furthermore, for three sizes with the same 200 void counts, increasing the bin number from 5 to 20 reduces the individual unfolding error from $5.4 \%$ to $1.2 \%$. These observations highlight that the unfolding accuracy can be considerably affected by factors such as void population and bin number, but not void size.

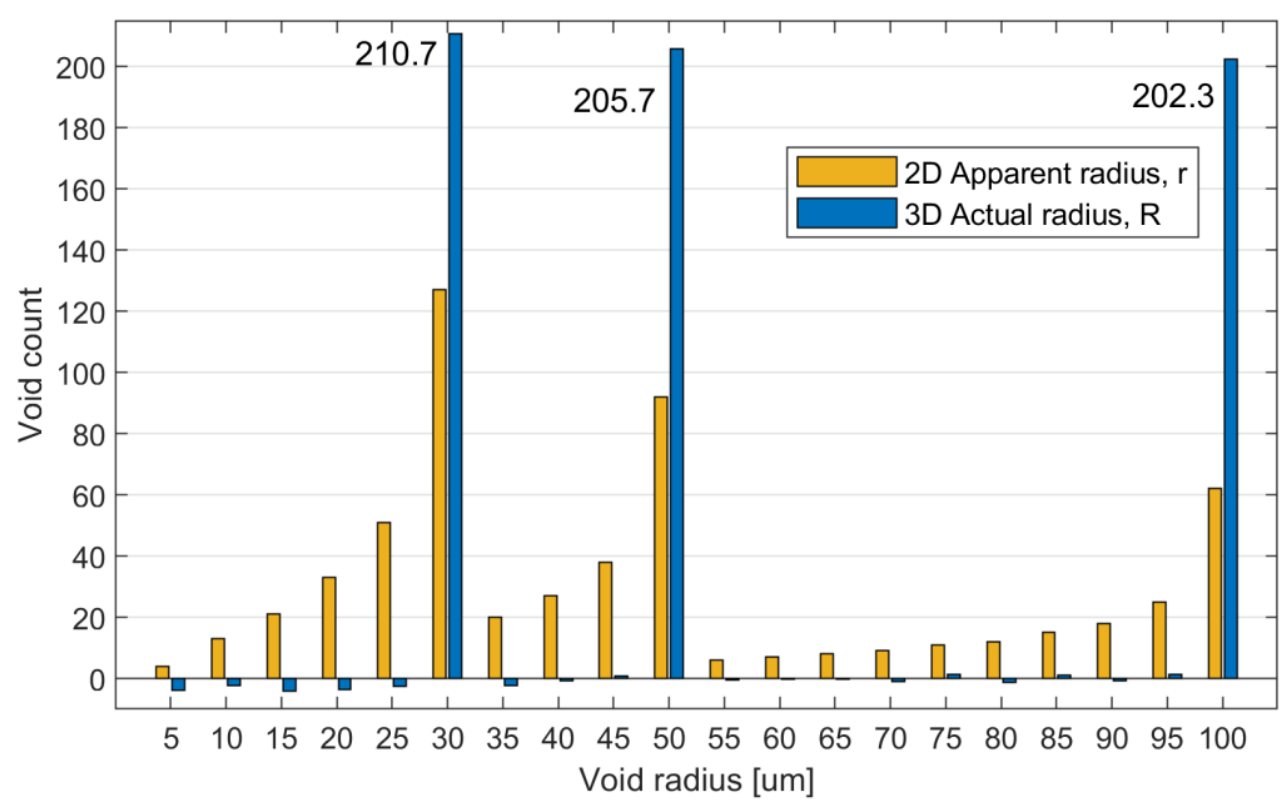

Fig. 3: Unfolding an idealized multi-sized void system $(\mathrm{R}=30,50$, and $100 \mu \mathrm{m})$ that emulates a simplified case of two hundred entrained air voids for each of the assumed size being randomly intersected on a polished concrete surface. The overall unfolding error is determined as $3.1 \%$.

To obtain the optimal unfolding strategy, it is pertinent to further clarify the influence of the various factors on the accuracy when varying continuously. To address this problem, the combined effect on altering both the void population and bin number in a mono-sized void system of 100- 
$\mu \mathrm{m}$ spheres is further investigated and displayed in Fig. 4. Based on this analysis, the void population is of special importance in this case, as a larger population almost always results in a lower unfolding error. As for bin number, the trend is more complex - the benefit of increasing bin number is attained only when enough voids are being considered. Under a deficient void count of 100, the unfolding error fluctuates as a function of bin number, which should be related to the periodicity of the round-off error. Although it is difficult to get an analytical solution for the optimal bin number, the determination of the bin number should certainly be made according to the void population.

While minimizing error can be well understood in the case of idealized void systems, complexity arises from the unfolding technique implemented with concrete. In particular, the difficulty arises regarding the effect of varying air voids bought by the different levels of air entrainment; other concerns involve how many scans are needed for obtaining a reasonable prediction of the 3D void distributions in these concretes. These concerns are addressed in the next section with real concrete scans.

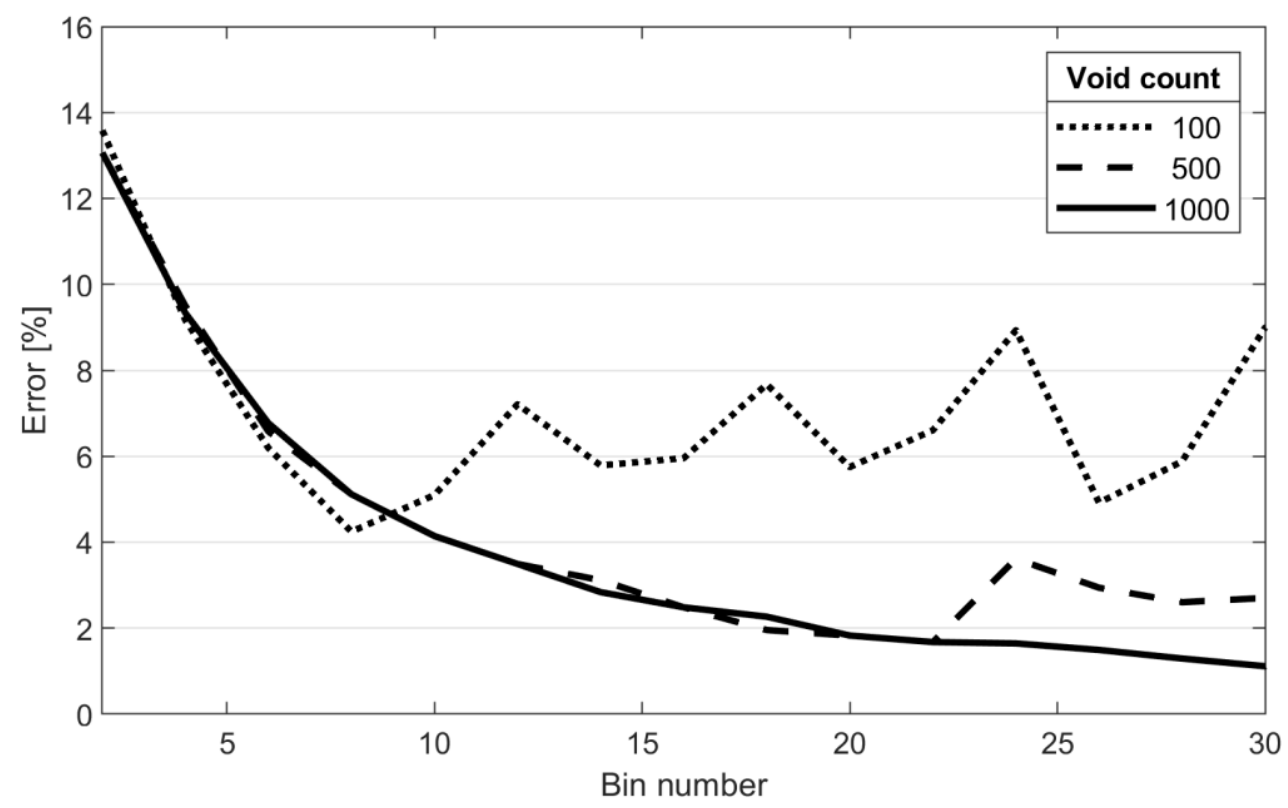

Fig. 4: Influence of void population and bin number on the unfolding error in a mono-sized void system $(\mathrm{R}=100 \mu \mathrm{m})$ that emulates a simplified case of entrained air voids of this size being randomly intersected on a polished concrete surface.

\section{Unfolding concrete void systems}

The air void system in concrete can be deemed as a complex multi-sized void system, which can still be deconvoluted using the unfolding technique. A conceptual concrete void size distribution 
is illustrated in Fig. 5, where the total height of stacked bins reflects the 2D apparent void size distribution. In each of the bin, the colored sections correspond to the $3 \mathrm{D}$ actual voids yielding a $2 \mathrm{D}$ intersection at this bin, as obtained after unfolding. The general steps for deconvoluting such a $2 \mathrm{D}$ void size distribution is detailed as follows. On a polished concrete section, the largest void intersections only originate from the largest voids, the 2D void size distribution can be unfolded sequentially from the largest to the smallest voids. As the $500-\mu \mathrm{m}$ voids can be cut at different positions, the yielded intersections will have apparent radii on the $2 \mathrm{D}$ plane that also fall into all the smaller sized bins. The resultant allocation to each bin follows the distribution defined by Eq. 1. In this case, this is highlighted by the dark red columns in Fig. 5; in turn, the counts of these sub-bins contribute to the actual population of the 500- $\mu \mathrm{m}$ spheres. After storing the total count of the $500-\mu \mathrm{m}$ voids and deducting them from the overall distribution, the next $450-\mu \mathrm{m}$ size bin, now the largest, is unfolded in the same manner. This processing loop is repeated until the smallest size bin is reached, whereby the whole $2 \mathrm{D}$ size distribution is unfolded from $2 \mathrm{D}$ to $3 \mathrm{D}$.

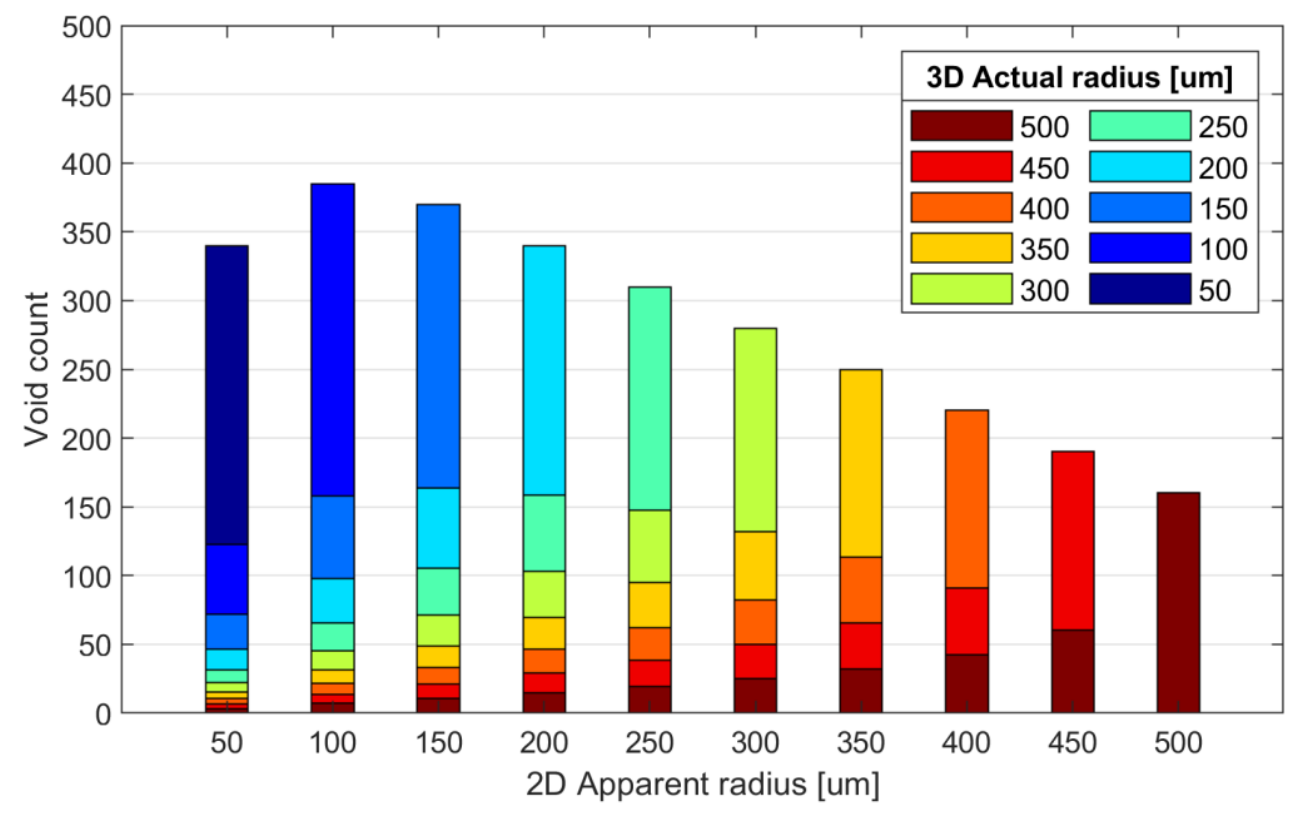

Fig. 5: Conceptual illustration of the 3D actual void sizes embedded in a 2D apparent void size distribution. The 2D distribution depicts a series of sphere intersections observed on a plane.

However, unfolding the actual void intersections that appeared in a concrete image involves more complexity. This is mainly because the real concrete voids are not always ideal in terms of the void shape, uneven size distribution, as well as image resolution (this is, the smallest voids might be pixelized so that their shapes are distorted). Furthermore, there is a trade-off between satisfying a robust statistical measurement versus ensuring a sufficiently high resolution for resolving all the entrained air voids. Due to this problem, it can be time-consuming to prepare the samples and 
conduct the measurement at a large sampling volume with good representativeness. Another challenge here lies in the validation of the unfolded size distribution, which requires measuring a reference of the actual void size distribution in concrete. In this regard, the CT investigations on the 3D concrete air void system are typically limited to a millimeter-level scale and most of the CT samples do not comprise of coarse aggregates [16,17,30,31]. Therefore, a stereological model is proposed to emulate $3 \mathrm{D}$ voids being intersected over a large area of concrete, based on real concrete scan images. The use of this model allows for the investigation of both 2D and 3D size distributions of the intersected voids, which provides a feasible way to evaluate the unfolding accuracy. Further details about the stereological model are provided in Section 4.3.

Herein, the reliability of the unfolding algorithm on real concrete is assessed with three groups of concrete mixtures that are non-, moderately-, and overly-air-entrained. The work can be divided into four phases, as illustrated in Fig. 6. The first phase comprises sample preparation and image segmentation. The second phase focuses on obtaining the 3D void size distribution from the void intersections that appeared in the image segmentation. During this step, the different binning schemes are additionally studied to better fit the characteristic of the concrete void size distribution. The third phase involves generating the stereological model. This model serves as a proxy of the intersected 3D air voids on the concrete section. As the final step, the goal of the fourth phase is evaluating the unfolding accuracy for real concrete. This is done by i) extracting the new 2D section from the stereological model, ii) obtaining its $2 \mathrm{D}$ and $3 \mathrm{D}$ void size distribution, and iii) comparing the newly unfolded 3D size distribution with the voids in the stereological model (whose 3D size distribution is known). Thus, the unfolding accuracy on real concrete can be measured based on the agreement on the 3D size distributions. The details of each phase are addressed by the following subsections.

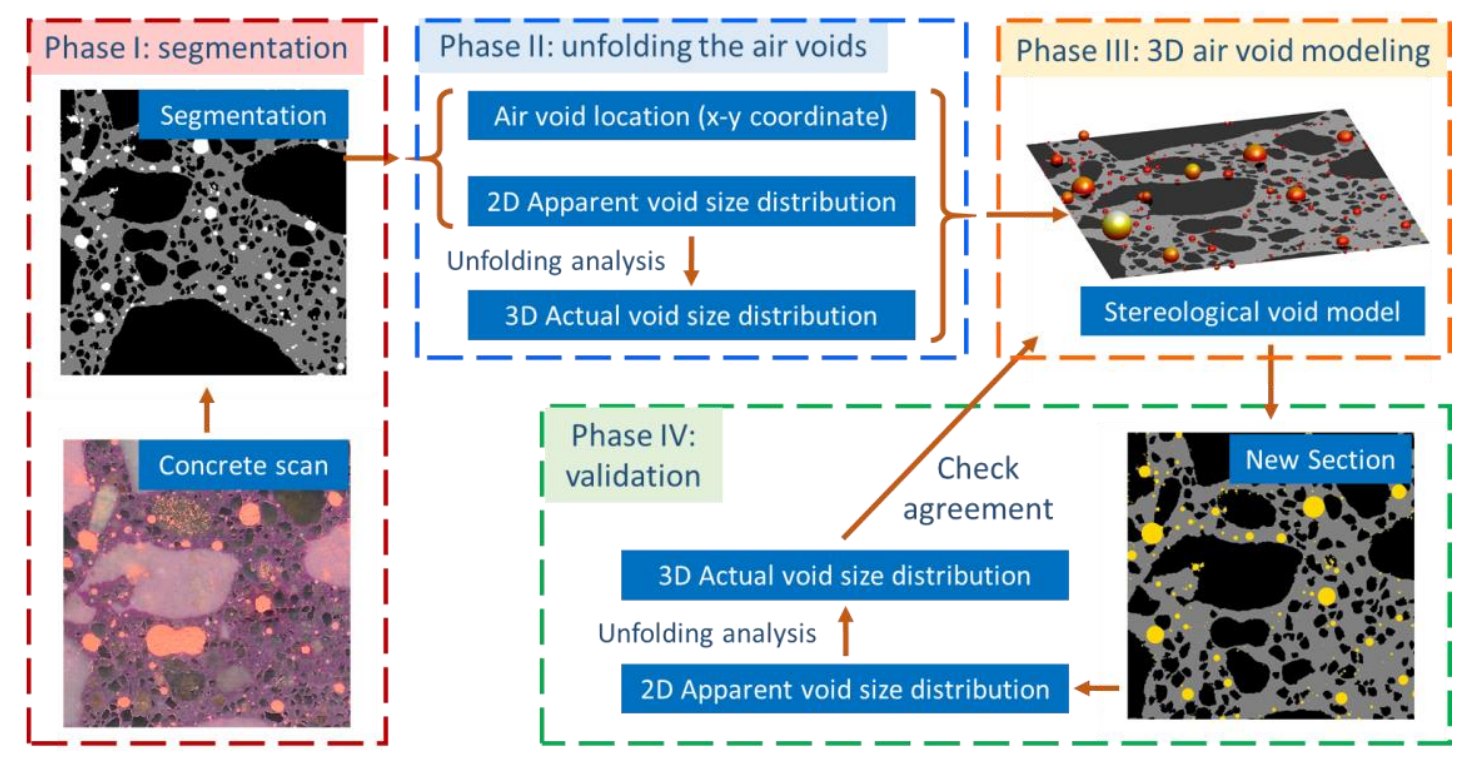

Fig. 6: Outline of the workflow for unfolding the concrete void size distribution. 


\subsection{Experimental work}

Three concrete mixtures with different air entrainment were cast to yield real concrete void system for the unfolding analysis. To make the level of air entrainment as the single variable, these mixtures were sampled from a single batch of fresh concrete mix. This base mix was made of $398.3 \mathrm{~kg} / \mathrm{m}^{3}$ cement (Essroc Italcementi Group, Type I/II), $943.1 \mathrm{~kg} / \mathrm{m}^{3}$ limestone (Illini Brick, 4.75 to $12.70 \mathrm{~mm}$ ), $763.5 \mathrm{~kg} / \mathrm{m}^{3}$ river sand (Illini Brick, passing $4.75-\mathrm{mm}$ sieve), and $179.5 \mathrm{~kg} / \mathrm{m}^{3}$ water. After mixing, a portion of the batch mix was cast into a $100 \times 200-\mathrm{mm}$ standard cylinder, as non-air-entrained "NA". Meanwhile, the remaining batch was air-entrained and further mixed for 60 seconds. The air-entraining admixture (AEA) used was DARAVAIR® 1400, and the equivalent dosage was $74.5 \mathrm{ml} / \mathrm{m}^{3}$ for the volume left, following the manufacturer's recommendation. The second cylinder was then sampled, as moderately air-entrained "MA". Afterward, the batch left was further air-entrained with $296.3 \mathrm{ml} / \mathrm{m}^{3}$ AEA and mixed for another 60 seconds. The third sample collected was as overly air-entrained "OA". All the fresh concrete cylinders were vibrated for 60 seconds, allowed for hardening under a sealed condition for 24 hours, and then demolded.

After 28-day moist curing, each cylinder was sawed longitudinally. The surface was then progressively polished using $250,110,70,30,15$, and $3-\mu \mathrm{m}$ disc, processed with surface treatments to contrast the cement paste using a phenolphthalein solution (5\% by weight in 200 proof alcohol), and then the air voids were filled using an orange chalk powder. The detailed steps of surface preparation are justified to be successful and reported in the literature [14,32]. Once done, five $50 \times 50 \mathrm{~mm}$ regions from each mixture were scanned using a flatbed scanner at a $5.3 \mu \mathrm{m}$ pixel resolution. The nomenclature for the individual sample image is "mixture type-scan number", such as "MA-3".

Based on the color contrast rendered from the surface treatments, the air void, paste, and aggregate phases in the concrete images were then segmented using Multispec and ImageJ [14]. During the image processing, the in-aggregate voids that are non-relevant to the concrete freeze-thaw behavior were removed. An example of the scanned image and its segmentation of each mixture are displayed in Fig. 7. In subsequent analysis, only the void intersections of an equivalent radius between 10 to $500 \mu \mathrm{m}$ were recorded. This is done because a void smaller than this range is not likely to alter the concrete freeze-thaw performance [14,33,34], while the irregular-shaped voids larger than this range have a negligible effect $[35,36]$. Furthermore, the small air voids falling into this range (especially the entrained voids) tend to maintain a spherical shape due to surface tension, as well as less physical constraint. Based on the collected statistics, the air content and total void count for each mixture were given in Table 1, where both parameters increase with the level of air entrainment. 

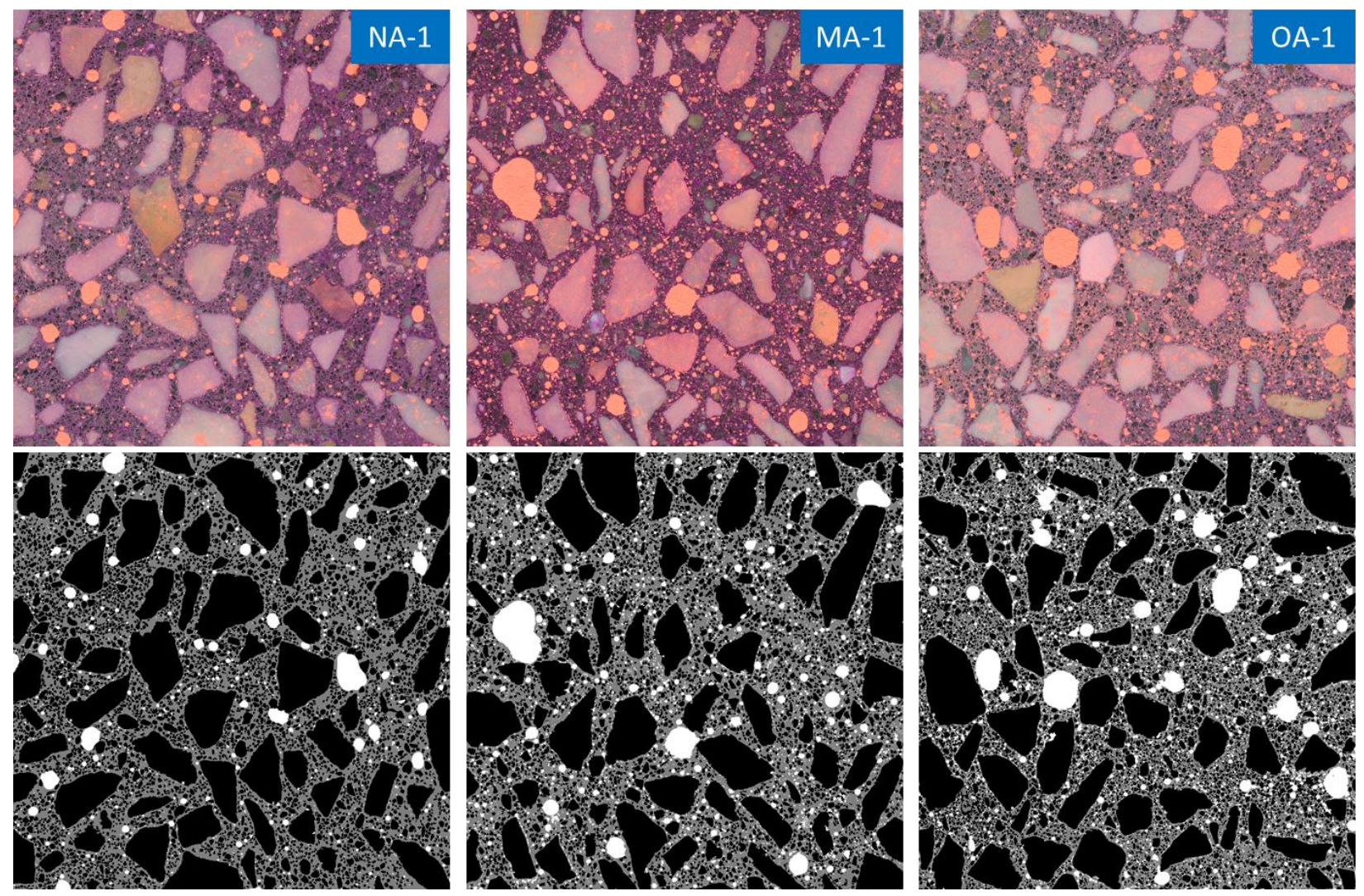

Fig. 7: The $50 \times 50 \mathrm{~mm}$ scan images for samples NA-1, MA-1, and OA-1, along with their threephase images after segmentation.

Table 1: The void content and void count of the three concrete mixtures. The standard deviation is calculated based on the five individual measurements.

\begin{tabular}{|c|c|c|c|}
\hline & Mixture NA & Mixture MA & Mixture OA \\
\hline Air content [\%] & $2.53 \pm 0.30$ & $5.88 \pm 0.15$ & $8.65 \pm 1.18$ \\
\hline Total void count & $3439 \pm 767$ & $14063 \pm 784$ & $22131 \pm 462$ \\
\hline
\end{tabular}

3.2 Unfolding the concrete void system using an optimized binning strategy

A major difference between the concrete void system and an idealized multi-sized void system (e.g. the one displayed in Fig. 3) comes from the fact that the population of air voids varies drastically with the void size, which constitutes a hindrance to the unfolding analysis. In particular, concrete void size distribution follows a lognormal trend [27,30,37] — this is, most of the voids concentrate at the small size while the population of large voids is extremely low, as governed by physical laws [38]. Based on the findings from Section 2.2, the unfolding accuracy is readily affected by the least populated voids. Hence, it is of special importance to avoid extremely small 
void counts in the large-sized bins for unfolding concrete air voids. In concrete studies, the void size distribution is conventionally presented using evenly-spaced bins (linear binning) [27,28,31,39]. Following this fashion, the $2 \mathrm{D}$ void size distributions of the three concrete mixtures are shown in Fig. 8a, where the twenty bins are used to ensure a reasonable resolution across the entire size range. It can be noted that the size distribution for these three mixtures are all extremely uneven, where the void population is different by several orders of magnitude between the two ends. The problem of linear binning is that it leads to a distribution extremely populated at a few single small-sized bins. Furthermore, there are only six bins located between 10-150 $\mu \mathrm{m}$ radius, where are dominated by entrained air voids [1,2]. This small bin number will greatly cap the unfolding accuracy, regardless of the void population. For example, based on Fig. 4, the unfolding error with six bins is at around 7\% even the void population increases from 100 to 1000 . For all the above reasons, it would be still challenging to address the problem of unfolding real concrete voids, even with additional sampling.

In this regard, a possible solution lies in altering the binning strategy. Specifically, the logarithmic binning provides a better representation of the lognormal distribution of concrete air voids [25]. This is because logarithmic binning, as compared to linear binning, enlarges the bin spacing for the large voids and reduces that for the small voids. As an illustration, the $2 \mathrm{D}$ void size distributions of the three concrete mixtures are revisited using logarithmic binning in Fig. 8b. As expected, the new 2D size distributions are more even. Furthermore, the number of bins between $10-150 \mu \mathrm{m}$ radius is improved from four (with linear binning) to fourteen (with logarithmic binning), which significantly eases and improves the unfolding analysis. Note that these improvements are attained without additional sampling. The implication of these changes to the unfolding outcome is discussed next. 

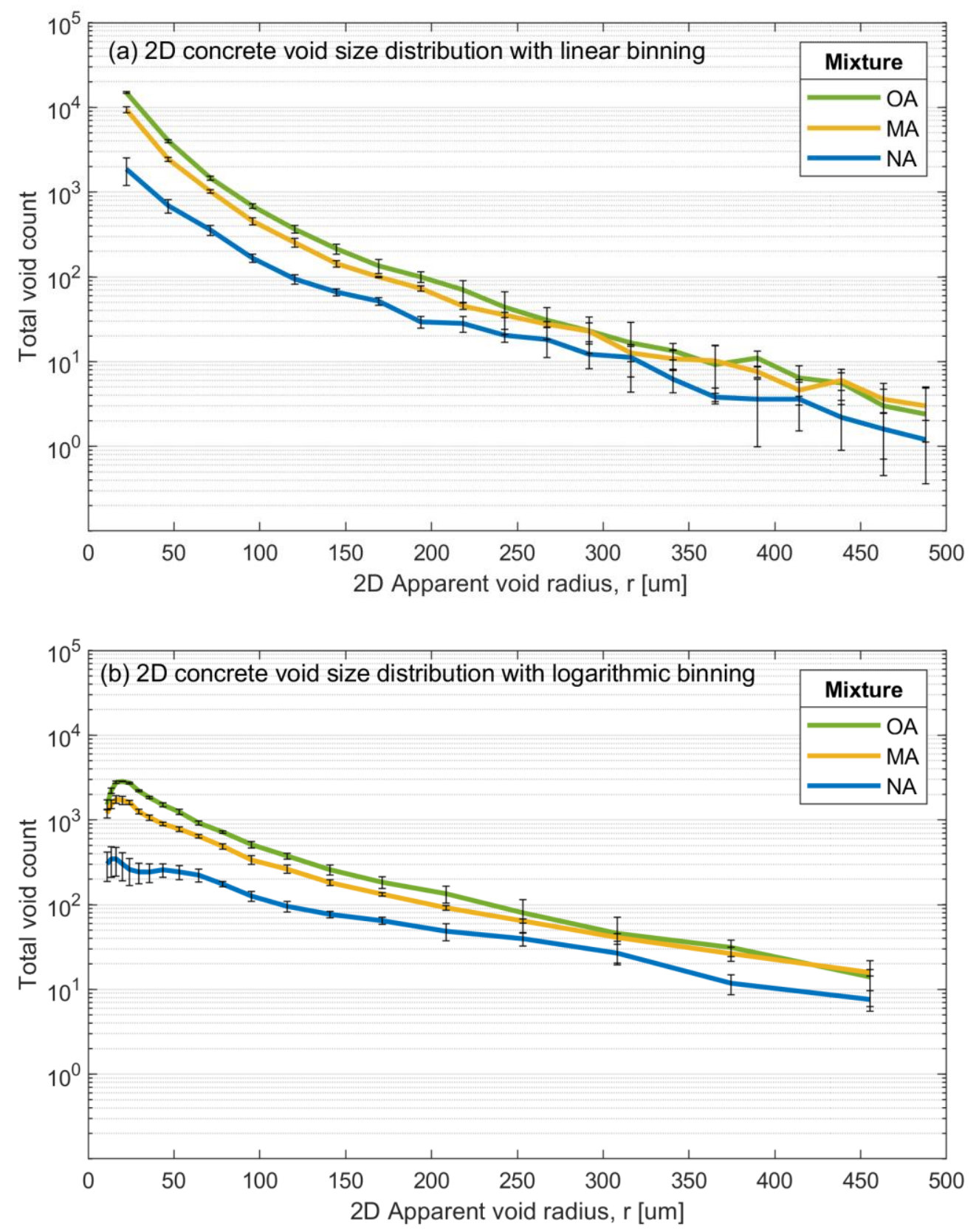

Fig. 8: The mean 2D apparent void size distributions for each of the three concrete mixtures, as obtained with (a) linear binning scheme and (b) logarithmic binning scheme. The error bars show the standard deviations of the five individual measurements.

The linear and logarithmic 2D distributions are then unfolded to restore their corresponding 3D void size distributions, as displayed in Fig. 9. As the concrete void size is supposed to be lognormally distributed, the unfolded distributions are then fitted in this format, as shown with the red curves. In this regard, the contrast between linear and logarithmic binning is quite self- 
explaining. The fittings for linear binning are highly skewed. There are only a few bins describing the small voids, and the void population vanishes quickly as the void size increases. Since the concrete freeze-thaw performance is largely controlled by the small entrained air voids, resolving the $3 \mathrm{D}$ void size distribution at the lower sizes is critical for evaluating the real concrete void system and its influence on concrete durability. Hence, the information provided by linear binning is rather limited. In contrast, the logarithmic binning in Fig. 9b provides a fine description of the size distribution of the small voids whilst substantially more counts are allocated to the larger bins. The use of logarithmic binning helps to address the small voids in a much proper way, resulting in a finer description of the concrete voids. Based on this comparison, only the logarithmic binning is further investigated in the subsequent sections. 

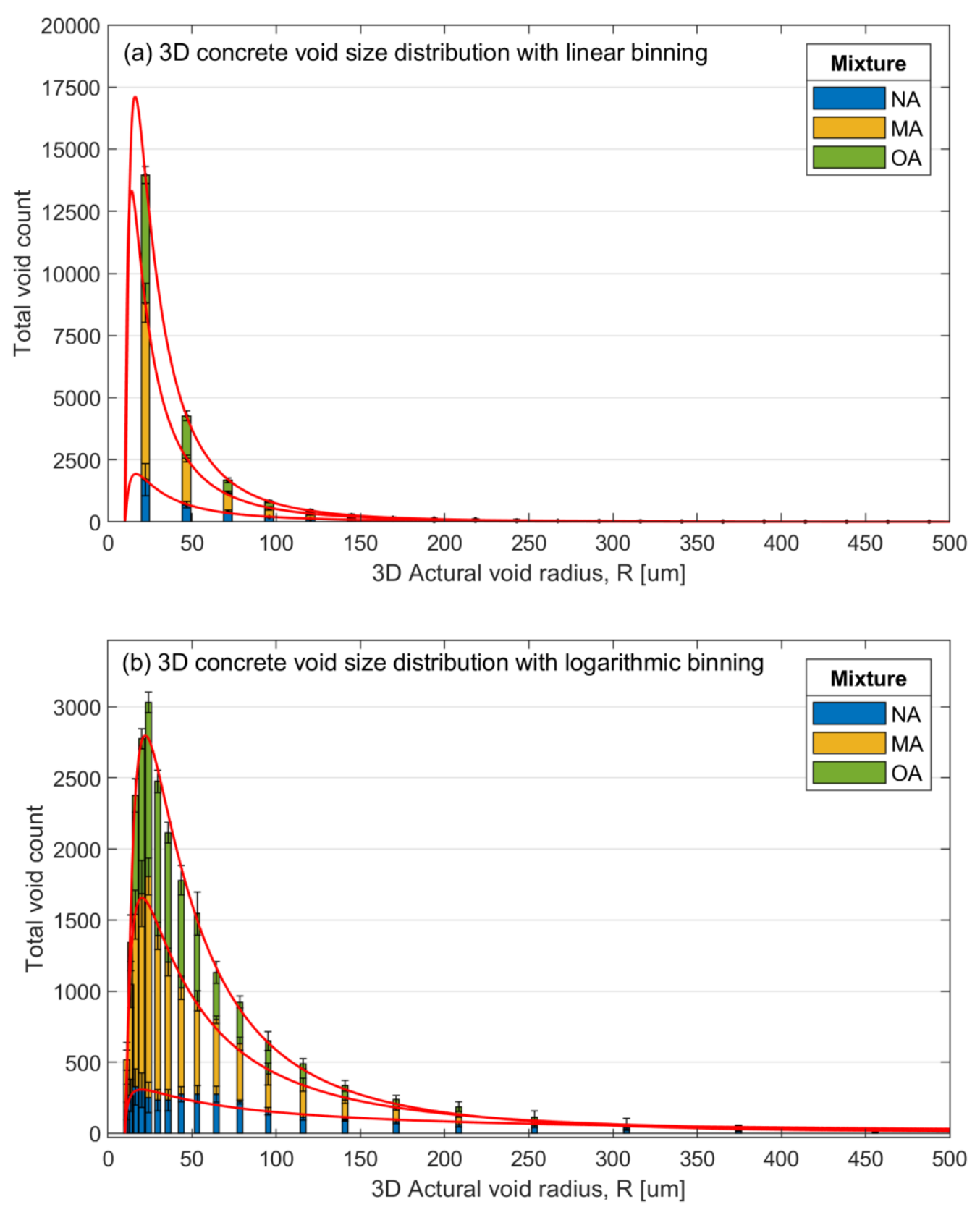

Fig. 9: The 3D void size distributions restored from the 2D void intersections observed on the concrete sections, with (a) linear binning and (b) logarithmic binning. The red curves show the lognormal fitting for each mixture. Note that the bottom sections of the color bars are overlapped and the short sections in each bin are not stacked. 


\subsection{Stereological modeling of the air voids in concrete}

To enable the validation of the unfolding analysis for real concrete, a stereological model was generated to emulate the $3 \mathrm{D}$ air voids being intersected on the $2 \mathrm{D}$ concrete section. The modeling inputs are i) the $x-y$ coordinate of the centroid of each void, ii) the 2D size distribution, and iii) the 3D size distribution. As explained at the beginning of Section 3, the modeling generates 3D spheres as the air voids from the largest actual size to the smallest. Taking the largest-sized voids as an example, the actual sizes of the spheres are identical, and their apparent sizes can be calculated proportionally using Eq. 1, whereby the z-coordinate (i.e., the height of the void centroid; either positive or negative) of each sphere is prescribed. Subsequently, the spheres are assigned to the concrete image, to the void intersections with matched apparent sizes. In case that there are more suitable locations, the sphere assignment is carried out stochastically. After generating all the largest voids, the above procedures are reiterated for the next bin size. During the entire modeling process, any newly generated void that overlapped with the existing ones is reattempted elsewhere.

Despite there is some uncertainty involved during modeling, the overall void assignment takes a statistical significance, allowing the model to serve as a reasonable approximation for the real voids being intersected. As a demonstration, the 3D models of the three images shown in Fig. 7 are displayed in Fig. 10. For visualization, these models show the $10 \times 10 \mathrm{~mm}$ upper left portion of the original images. Beneath the model shows of the new void intersections yielded. Note that the void intersections shown in light gray have an equivalent radius larger than $500 \mu \mathrm{m}$.

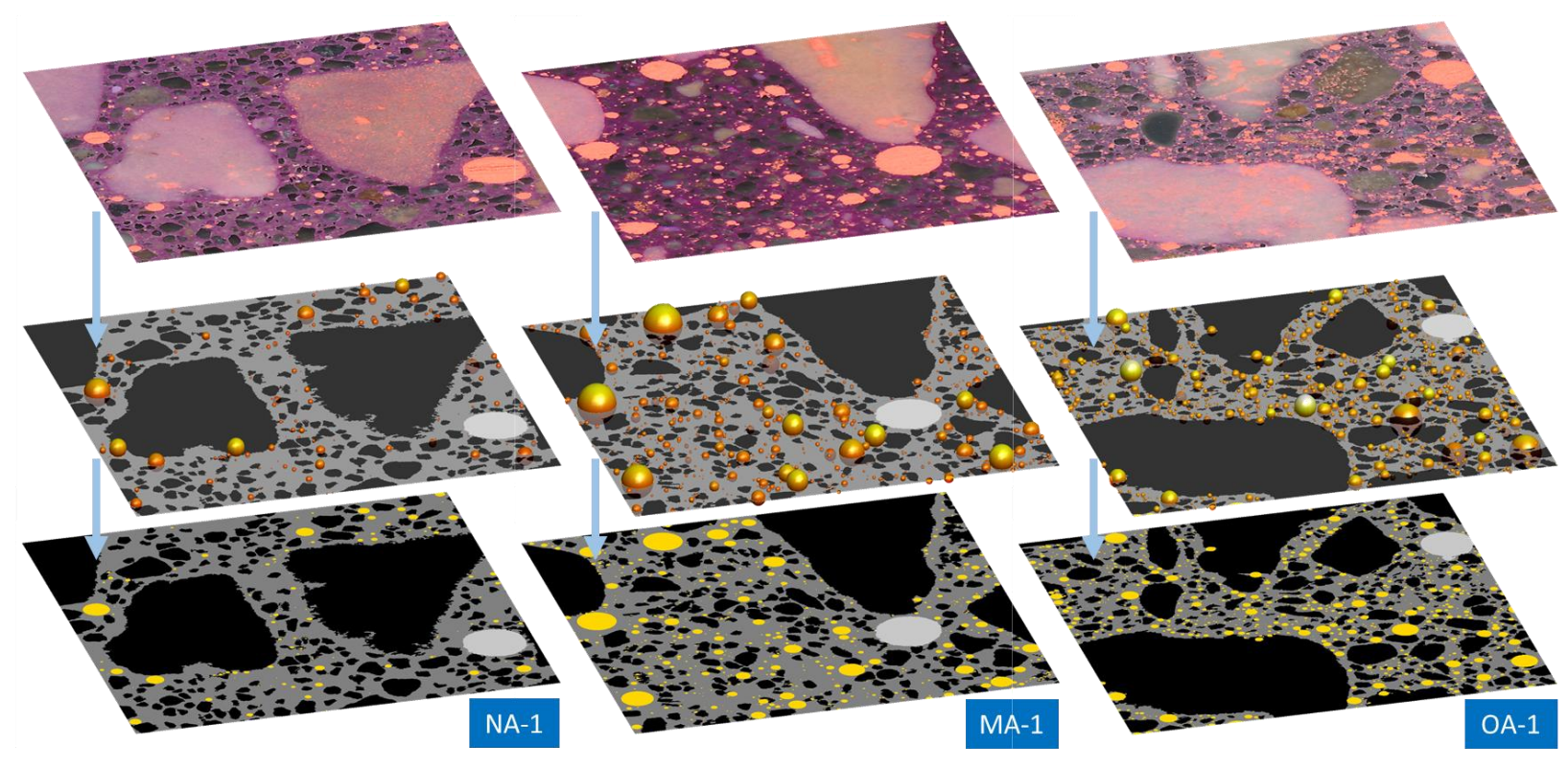

Fig. 10: Stereological modeling of the voids in NA-1, MA-1, and OA-1, with the concrete images (top), the models generated (middle), and the new intersections yielded (bottom). For visualization, only the $10 \times 10 \mathrm{~mm}$ upper left corners of the original scans are displayed. 


\subsection{Validation of the unfolding analysis for concrete}

To evaluate the accuracy of the unfolding analysis for concrete, the void intersections extracted from the 2D model section (e.g., the bottom images displayed in Fig. 10) are unfolded and compared with the actual 3D void size distribution of the model. The processing generally shares the same procedures as described in Section 3.2. Note that the virtual $2 \mathrm{D}$ concrete section is the only input of this evaluation. This is the predicted 3D void size distribution in this step is obtained without any knowledge of the original 3D void sizes in the stereological model.

The predicted 3D void size distributions are displayed in Fig. 11. Comparing to the original size distributions (see Fig. 9b), the predicted ones are almost identical for each mixture, as also supported by the well-matched lognormal fittings. The unfolding error for the concrete mixtures is calculated using Eq. 3 and the results are displayed in Table 2. Here, to decompose the overall unfolding error, the air voids are further divided into two groups representing the radius range dominated by the entrained air $(10-150 \mu \mathrm{m})$ and by the entrapped air $(150-500 \mu \mathrm{m})$ [1,2]. Note that the step point here only serves as a reasonable split between the regions occupied by entrained and entrapped air, but not a solid threshold. The unfolding errors associated with the smaller air voids are more affected by the air entrainment than those of the larger voids across all the mixtures. This is expected, as the air-entraining agent mainly acts on the small air bubbles [38]. It can be also noticed that the overall unfolding error is largely controlled by that of the smaller voids (where the air voids are more populated). In contrast, the unfolding error of the larger voids remains relatively stable, unless an excessively high dosage of the air-entraining agent is used (i.e., Mixture). Although the small voids are last processed during the unfolding analysis, the potential unfolding accuracy loss is partially compensated by the superior void population than the large ones, especially for the air-entrained mixtures.

In terms of the overall error, it decreases significantly at greater air entrainment. Based on the investigation in Section 2.2, this change should be primarily attributed to the increased void population at small sizes. Among the three cases, the largest overall unfolding error is found in Mixture NA as 4.32\%; even for this non-air-entrained concrete, the unfolding error is not significant. According to the correlation between the overall unfolding error and the total void number (see Table 1), a minimum of 2500 voids seems to be a reasonable requirement to ensure a reliable unfolding analysis of around 5\% error. It should be noted that the above observations may vary when the key attribute to the concrete air void systems are changed, such as type of airentraining admixture or the use of other admixtures exhibiting a detraining effect. Although it is not likely that their impact can fundamentally change void size distribution in concrete, it is worth to repeat this kind of validation on the new samples to ensure a high quality of the unfolding analysis. 


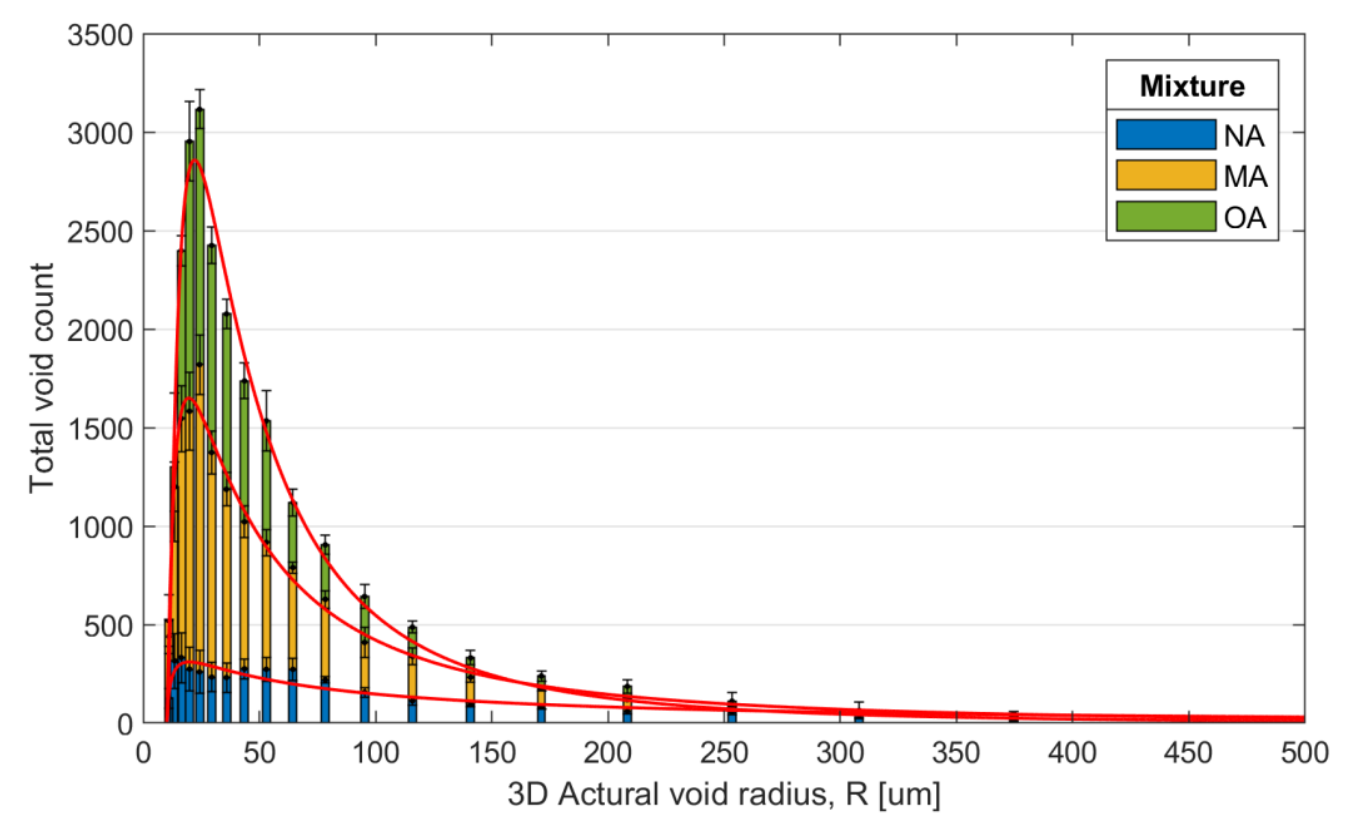

Fig. 11: The restored 3D void size distributions of the voids in the stereological models, where the restoration is accomplished with the sole knowledge of the 2D intersections extracted from these models. These 2D intersections surrogate the concrete images for hardened concrete void analysis. Note that the bottom sections of the color bars are overlapped and the short sections in each bin are not stacked.

Table 2: The sectional and overall unfolding error of the three concrete mixtures. For each mixture, the results are calculated from five individual measurements.

\begin{tabular}{|c|c|c|c|}
\hline \multirow{2}{*}{ Mixture } & \multicolumn{2}{|c|}{ Sectional unfolding error [\%] } & \multirow{2}{*}{$\begin{array}{c}\text { Overall unfolding error } \\
{[\%]}\end{array}$} \\
\hline & $\begin{array}{c}10-150 \mu \mathrm{m} \\
(\text { mostly entrained air) }\end{array}$ & $\begin{array}{c}150-500 \mu \mathrm{m} \\
(\text { mostly entrapped air })\end{array}$ & \\
\hline NA & $4.51 \pm 1.07$ & $1.93 \pm 0.14$ & $4.32 \pm 0.98$ \\
\hline MA & $3.18 \pm 0.57$ & $1.93 \pm 0.15$ & $3.13 \pm 0.54$ \\
\hline $\mathrm{OA}$ & $2.67 \pm 0.20$ & $0.67 \pm 0.05$ & $2.62 \pm 0.19$ \\
\hline
\end{tabular}

\section{Conclusions}

Information on the 3D concrete void system is of special importance for improving understanding of concrete freeze-thaw behavior. However, the 2D void size distribution directly measured from a polished concrete section does not represent the actual void size distribution in $3 \mathrm{D}$. To solve this problem, we investigated the use of the 2D-to-3D unfolding technique on concrete void systems. 
In particular, this study aims at determining the optimal unfolding strategy for a given concrete scan.

From the investigation on the idealized void systems, the results revealed that that the unfolding accuracy is greatly influenced by the void population, which is fundamental to satisfy the probabilistic assumptions of the unfolding algorithm. With a large void population coupled with sufficient bins, proper representation of the 3D size distribution of concrete voids is attainable. The subsequent investigation on the real concrete samples highlights that logarithmic binning serves as a superior choice to reflect the lognormally distributed concrete void systems, versus the conventionally used linear binning. This is mainly because logarithmic binning can provide a finer description regarding the size information of the small voids, which are directly associated with concrete freeze-thaw performance. To overcome the difficulties of obtaining the actual void size distribution of the concrete mixtures, we proposed a stereological void model to emulate the actual air voids being intersected on the concrete section. Based on the unfolding result for this model, the unfolding void size distribution matches well with the true distribution that is known. The unfolding accuracy for real concrete is found to increase with greater air entrainment, while the unfolding error is still reasonably low for non-air-entrained concrete $(<5 \%)$.

Overall, these findings are encouraging as they suggest high confidence in studying the concrete air void system with the proposed unfolding strategy. Despite the various air entrainment in concrete, choosing the right binning strategy and maintaining a certain void population are critical for a reliable unfolding analysis. For optimal concrete unfolding analysis, it is recommended to i) use logarithmic binning, and ii) ensure a minimum of 2500 voids to be considered for each concrete sample. With these, the accurate unfolding can offer new possibilities to improve the future concrete air void analysis to a $3 \mathrm{D}$ level.

\section{References}

[1] J. Szwabowski, B. Lazniewska-Piekarczyk, Air-entrainment problem in self-compacting concrete, J. Civ. Eng. Manag. (2009). https://doi.org/10.3846/1392-3730.2009.15.137-147.

[2] S. Rath, N. Puthipad, A. Attachaiyawuth, M. Ouchi, Critical Size of Entrained Air to Stability of Air Volume in Mortar of Self-Compacting Concrete at Fresh Stage, J. Adv. Concr. Technol. 15 (2017) 29-37. https://doi.org/10.3151/jact.15.29.

[3] H.S. Wong, A.M. Pappas, R.W. Zimmerman, N.R. Buenfeld, Effect of entrained air voids on the microstructure and mass transport properties of concrete, Cem. Concr. Res. (2011). https://doi.org/10.1016/j.cemconres.2011.06.013.

[4] C. Powers, T, A. Helmuth, R, THEORY OF VOLUME CHANGES IN HARDENED PORTLANDCEMENT PASTE DURING FREEZING, in: Proc. Thirty-Second Annu. Meet. Highw. Res. Board, 1953: pp. 285-297.

[5] T. Powers, T. Willis, The air requirement of frost resistant concrete, in: Proc. Twenty-Ninth Annu. Meet. Highw. Res. Board Held Washington, D.C. December 13-16, 1949., 1950: pp. 184-211.

[6] K.C. Hover, Some Recent Problems with Air-Entrained Concrete, Cem. Concr. Aggregates. 11 (1989) $67-$ 72. 
[7] T.D. Hanson, J. Hart, Plastic Air Content versus Hardened Air Content by High Pressure Air Meter Of Transportation Plastic Air Content versus Hardened Air Content by High Pressure Air Meter, 2001.

[8] Astm C 457, Standard Test Method for Microscopical Determination of Parameters of the Air-Void System in Hardened Concrete 1, ASTM Int. 05 (2013) 1-15. https://doi.org/10.1520/C0457.

[9] S. Chatterji, H. Gudmundsson, Characterization of entrained air bubble systems in concretes by means of an image analysing microscope, Cem. Concr. Res. 7 (1977) 423-428. https://doi.org/10.1016/00088846(77)90070-9.

[10] K. Peterson, R. Swartz, Air Void analysis of hardened concrete with a flatbed scanner, J. .... (2001) 1-28. https://doi.org/10.3141/1775-06.

[11] Z. Zhang, F. Ansari, N. Vitillo, Automated determination of entrained air-void parameters in hardened concrete, ACI Mater. J. 102 (2005) 42-48.

[12] U.H. Jakobsen, C. Pade, N. Thaulow, D. Brown, S. Sahu, O. Magnusson, S. De Buck, G. De Schutter, Automated air void analysis of hardened concrete - a Round Robin study, Cem. Concr. Res. 36 (2006) 1444-1452. https://doi.org/10.1016/j.cemconres.2006.03.005.

[13] K. Peterson, J. Carlson, L. Sutter, T. Van Dam, Methods for threshold optimization for images collected from contrast enhanced concrete surfaces for air-void system characterization, Mater. Charact. 60 (2009) 710-715. https://doi.org/10.1016/j.matchar.2008.10.005.

[14] Y. Song, R. Zou, D.I. Castaneda, K.A. Riding, D.A. Lange, Advances in Measuring Air-Void Parameters in Hardened Concrete Using a Flatbed Scanner, J. Test. Eval. 45 (2017). https://doi.org/10.1520/JTE20150424.

[15] R. Pleau, M. Pigeon, J.L. Laurencot, Some findings on the usefulness of image analysis for determining the characteristics of the air-void system on hardened concrete, Cem. Concr. Compos. 23 (2001) 237-246. https://doi.org/10.1016/S0958-9465(00)00079-2.

[16] E.N. Landis, D.J. Corr, THREE DIMENSIONAL ANALYSIS OF AIR VOID SYSTEMS IN CONCRETE, in: Meas. Monit. Model. Concr. Prop., Springer Netherlands, Dordrecht, n.d.: pp. 517-524. https://doi.org/10.1007/978-1-4020-5104-3_63.

[17] J. Schock, S. Liebl, K. Achterhold, F. Pfeiffer, Obtaining the spacing factor of microporous concrete using high-resolution Dual Energy X-ray Micro CT, Cem. Concr. Res. 89 (2016) 200-205. https://doi.org/10.1016/j.cemconres.2016.08.008.

[18] T.D. Larson, P.D. Cady, J.J. Malloy, The protected paste volume concept using new air-void measurement and distribution techniques, J. Mater. 2 (1967) 202-226.

[19] K.W. Peterson, R.A. Swartz, L.L. Sutter, T.J. Van Dam, Hardened Concrete Air Void Analysis with a Flatbed Scanner, Transp. Res. Rec. J. Transp. Res. Board. 1775 (2001) 36-43. https://doi.org/10.3141/177506.

[20] S.L. Peterson K., VanDam T., Determination of the Paste to Void Proximity Distribution in Two Dimensions from a Cross-Section through a Concrete Specimen, in: Eighth Euroseminar Microsc. Appl. to Build. Mater., 2001: pp. 4-7.

[21] J. Wawrzeńczyk, W. Kozak, A method of analyzing the porous microstructure in air-entrained concrete on the basis on 2D image analysis, in: Procedia Eng., 2015: pp. 102-107.

https://doi.org/10.1016/j.proeng.2015.06.124.

[22] J. Wawrzeńczyk, W. Kozak, Protected Paste Volume (PPV) as a parameter linking the air-pore structure in concrete with the frost resistance results, Constr. Build. Mater. 112 (2016) 360-365. https://doi.org/10.1016/j.conbuildmat.2016.02.196.

[23] D. Zalocha, J. Kasperkiewicz, Estimation of the structure of air entrained concrete using a flatbed scanner, Cem. Concr. Res. (2005). https://doi.org/10.1016/j.cemconres.2005.05.004.

[24] J.C. Russ, R.T. Dehoff, Practical Stereology, Springer US, Boston, MA, 2000. https://doi.org/10.1007/9781-4615-1233-2.

[25] E.J. Payton, Revisiting Sphere Unfolding Relationships for the Stereological Analysis of Segmented Digital 
Microstructure Images, J. Miner. Mater. Charact. Eng. 11 (2012) 221-242. https://doi.org/10.4236/jmmce.2012.113018.

[26] G. van Dalen, M.W. Koster, 2D \& 3D particle size analysis of micro-CT images, Bruker-MicroCT UserMeeting 2012. (2012) 16. http://bruker-microct.com/company/UM2012/31.pdf.

[27] P.C. Fonseca, G.W. Scherer, An image analysis procedure to quantify the air void system of mortar and concrete, Mater. Struct. Constr. 48 (2015) 3087-3098. https://doi.org/10.1617/s11527-014-0381-9.

[28] N.P. Mayercsik, R. Felice, M.T. Ley, K.E. Kurtis, A probabilistic technique for entrained air void analysis in hardened concrete, Cem. Concr. Res. 59 (2014) 16-23. https://doi.org/10.1016/j.cemconres.2014.01.023.

[29] S.A. Saltikov, The Determination of the Size Distribution of Particles in an Opaque Material from a Measurement of the Size Distribution of Their Sections, in: Stereology, Springer Berlin Heidelberg, Berlin, Heidelberg, 1967: pp. 163-173. https://doi.org/10.1007/978-3-642-88260-9_31.

[30] T.S. Yun, K.Y. Kim, J. Choo, D.H. Kang, Quantifying the distribution of paste-void spacing of hardened cement paste using X-ray computed tomography, Mater. Charact. 73 (2012) 137-143. https://doi.org/10.1016/j.matchar.2012.08.008.

[31] K.Y. Kim, T.S. Yun, J. Choo, D.H. Kang, H.S. Shin, Determination of air-void parameters of hardened cement-based materials using X-ray computed tomography, Constr. Build. Mater. 37 (2012) 93-101. https://doi.org/10.1016/j.conbuildmat.2012.07.012.

[32] Y. Song, A. Das, D.A. Lange, H. Mosavi, K. Riding, A Performance-Based Approach to Concrete FreezeThaw Durability, in: 2018 Jt. Rail Conf., ASME, 2018: p. V001T01A010. https://doi.org/10.1115/JRC20186167.

[33] J. Lamond, J. Pielert, Significance of Tests and Properties of Concrete and Concrete-Making Materials, ASTM International, 100 Barr Harbor Drive, PO Box C700, West Conshohocken, PA 19428-2959, 2006. https://doi.org/10.1520/STP169D-EB.

[34] K. Aligizaki, Pore Structure of Cement-Based Materials: Testing, Interpretation and Requirements, CRC Press, 2006.

[35] Z. Liu, W. Hansen, B. Meng, Characterisation of air-void systems in concrete, Mag. Concr. Res. (2015). https://doi.org/10.1680/macr.15.00074.

[36] W. Micah Hale, S.F. Freyne, B.W. Russell, Examining the frost resistance of high performance concrete, Constr. Build. Mater. (2009). https://doi.org/10.1016/j.conbuildmat.2008.04.006.

[37] K.A. Snyder, A Numerical test of air void spacing equations, Adv. Cem. Based Mater. 8 (1998) $28-44$. https://doi.org/10.1016/S1065-7355(98)00007-8.

[38] L. Du, K.J. Folliard, Mechanisms of air entrainment in concrete, Cem. Concr. Res. (2005). https://doi.org/10.1016/j.cemconres.2004.07.026.

[39] Z. Wang, Q. Zeng, L. Wang, K. Li, S. Xu, Y. Yao, Characterizing frost damages of concrete with flatbed scanner, Constr. Build. Mater. 102 (2016) 872-883. https://doi.org/10.1016/j.conbuildmat.2015.11.029. 